\title{
The Effect of Stress in the Density of States of Amorphous Carbon Films Determined by X-Ray Excited Auger Electron Spectroscopy
}

\author{
P. F. Barbieri ${ }^{1,2,3}$ and F. C. Marques $^{3}$ \\ ${ }^{1}$ Faculdade de Tecnologia “Arthur de Azevedo" (FATEC), Rod. Deputado Nagib Chaib 380, 13808-300 Mogi Mirim, SP, Brazil \\ ${ }^{2}$ Centro Universitário Adventista de São Paulo (UNASP), Campus de Engenheiro Coelho (EC), Estrada Municipal Pastor Walter Boger, \\ s/n, Lagoa Bonita, 13165-000 Engenheiro Coelho, SP, Brazil \\ ${ }^{3}$ Instituto de Física "Gleb Wataghin", Universidade Estadual de Campinas (UNICAMP), Caixa Postal 6165, Cidade Universitária, \\ 13083-970 Campinas, SP, Brazil \\ Correspondence should be addressed to F. C. Marques; marques@ifi.unicamp.br
}

Received 30 March 2017; Revised 23 June 2017; Accepted 11 July 2017; Published 8 August 2017

Academic Editor: David Holec

Copyright ( 2017 P. F. Barbieri and F. C. Marques. This is an open access article distributed under the Creative Commons Attribution License, which permits unrestricted use, distribution, and reproduction in any medium, provided the original work is properly cited.

\begin{abstract}
Amorphous carbon films can be prepared with a large variety of structure and have been used in a number of technological applications. Many of their properties have been determined, but very little is known concerning the effect of pressure on their properties. In this work we investigate the influence of pressure of graphite-like amorphous carbon films on the density of states (DOS) using X-ray Excited Auger Electron Spectroscopy (XAES) and the second derivate method of the XAES. The films were deposited by ion beam deposition and simultaneously bombarded with argon, which is responsible for the variation of the film stress, reaching extremely high values $(4.5 \mathrm{GPa})$. Marked variations of the density of states of the $p_{\pi}, p_{\sigma}$, sp, and s components were observed with increasing stress.
\end{abstract}

\section{Introduction}

Amorphous carbon films (a-C) have been investigated and technologically applied for decades due to their interesting properties such as high hardness, low friction coefficient, and chemical inertness or even as a matrix host for the implantation of other elements [1-3]. The ability of carbon to hybridize as $\mathrm{sp}^{1}, \mathrm{sp}^{2}$, and $\mathrm{sp}^{3}$ is the main reason for existence of a variety of different structures such as diamond, graphite, or even amorphous carbon $(\mathrm{a}-\mathrm{C})$. As for the latter one, the $\mathrm{sp}^{3} / \mathrm{sp}^{2}$ ratio has been used as a good parameter to determine its physical properties. It is well known that the $\mathrm{sp}^{3} / \mathrm{sp}^{2}$ ratio depends on the deposition method of the film and on the treatment to which it is subjected $[2,3]$.

Auger electron spectroscopy (AES) has been widely used in the investigation of carbon bonds in carbon based materials thin films because the main peak of the carbon Auger transition involves valence states [4-13]. The line shape of the
Auger spectrum is assumed to be a self-convolution of the DOS [10]; that is, the Auger electrons exhibit energy proportional to the occupied state band energy. This correspondence between DOS and carbon KVV spectra was observed and studied by Lascovich and coauthors [10], where the validity of the weak interaction between holes is the energy region next to the Fermi level ( $\pi$ band). On the other hand, some theoretical efforts have also been made in understanding the experimental Auger spectra, starting from known principles of electronic states $[14,15]$.

X-ray Excited Auger Electron Spectroscopy (XAES), on the other hand, seems to play a better role in the analysis of electronic structure, allowing more precise interpretations of the fine structure of the spectra. That is because, in the case of $\mathrm{AES}$, the electron beam damages the surface, broadening the spectral lines [4].

In the studies of carbon materials, employing analysis of KVV spectra, alternative methods have been proposed, such 
as the method of the first derivative and second derivative. With regard to the first method, it has been largely used to determine the energy and linewidth of specific orbitals, which are used to characterize the material. For instance, it has been shown that graphitic samples exhibit a width of $\sim 23 \mathrm{eV}[4,6]$.

The method of the second derivative presented by Lascovich and Santoni [6] has the advantage of showing in more detail the fine structures of the peaks corresponding to the original full spectrum; that is, to a first approximation, the minimum found in the second derivative of an Auger spectrum should correspond to a maximum of one full original peak. Making use of a simplified model (where the interactions between the final stage of holes are negligible), they showed that the KVV spectra reflect well the behavior of the DOS in comparison with theoretical data.

In this paper we studied a-C thin films using the second derivative method to determine, for the first time, the influence of the internal pressure (using the intrinsic stress) of the carbon matrix and how it affects the band states. The $\mathrm{a}-\mathrm{C}$ thin films were grown by IBAD (Ion Beam Assisted Deposition) in order to change the internal pressure of the carbon matrix through the implantation of noble gases in a similar fashion to that described in $[1,3]$. The samples exhibit high intrinsic stress, when compared to other techniques, and it is expected that the stress influences the most external electronic states, composed of s-p hybridized orbitals. Such influences can be perceived by spectroscopic techniques through changes in the line shape spectrum, the intensities and energy peaks, and peak-to-peak energies. For example, the line shape of the second derivative of the KVV spectrum of diamond is characteristic of the pure $\mathrm{sp}^{3}$-hybridized states tetrahedrally bonded ( $\sigma$-bonds) as well as that of graphite with line shape due to $\mathrm{sp}^{2}$ hybridization ( $\sigma$-bonds in plane and $\pi$-bonds normal to the $\sigma$-bond, for a threefold coordination). As for $\mathrm{a}-\mathrm{C}$ the line shape has contribution of both $\mathrm{sp}^{3}$ and $\mathrm{sp}^{2}$ hybridization [6], and evidently the density of states (DOS) of a-C is composed of $\sigma$ - and ${ }^{*} \sigma$-bonds and $\pi$ - $^{*} \pi$-bonds. However, the spectroscopy techniques normally investigate occupied states ( $\sigma$ and $\pi$ orbitals) of DOS in the valence band.

\section{Experimental}

The a-C films were deposited by IBAD (Ion Beam Assisted Deposition) using two Kauffman sources, one directed to the $\mathrm{C}$ target to perform sputtering, while the other source bombards the film during the growth process. Argon ions were used in both Kauffman sources. EELS measurements revealed that the films are graphite-like with $\mathrm{sp}^{2}$ concentration of approximately $90 \%$. The films were deposited on $\mathrm{n}$-doped $\mathrm{c}$-Si substrates $<111>4 \times 25 \times 0.4 \mathrm{~mm}^{3}$ at a temperature of $150^{\circ} \mathrm{C}$. The chamber pressure was maintained at $\sim 5 \times 10^{-4}$ mbar during deposition. The graphite target was bombarded with a constant bias of $-1500 \mathrm{~V}$, while the films were simultaneously bombarded with energy in the 0 to $-500 \mathrm{eV}$ range. Thicknesses of the films were in the 100-170 nm range. The samples were then transferred in situ to an UHV system (pressure of $\sim 7 \times 10^{-9}$ mbar) for XAES measurements. To collect the Auger spectra of the carbon

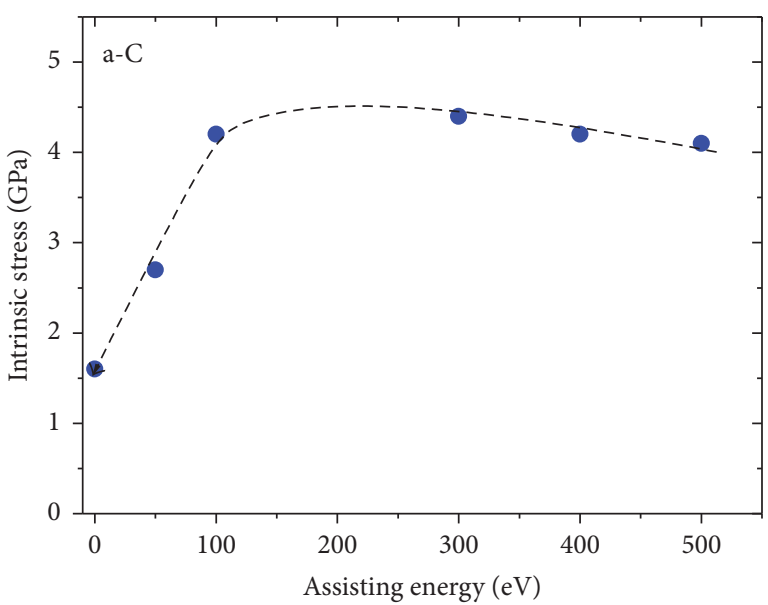

FIGURE 1: Compressive intrinsic stress as a function of the bombardment energy (assisting energy) with $\mathrm{Ar}^{+}$ions. The dashed line is a guide to the eyes.

atoms, the samples were excited with the $\mathrm{Al} \mathrm{K}_{\alpha} \mathrm{X}$-ray line $(1486.6 \mathrm{eV})$ and spectra were collected in the Fixed Analyzer Transmission (or FAT) mode with pass energy of $20 \mathrm{eV}$ and at a resolution of $0.6 \mathrm{eV}$.

After XAES measurements, the stress of the a-C films was measured using the "bending beam technique" using the radius of curvature of film/substrate composite obtained by the deflection of a He-Ne laser beam. The stress was then calculated using Stoney's equation (see details in [17]):

$$
\sigma=\frac{E}{(1-v)} \frac{t^{2}}{6 d}\left(\frac{1}{R}-\frac{1}{R_{0}}\right),
$$

where $E=229 \mathrm{GPa}, v=0.262$, and $t=0.41 \mathrm{~mm}$ are, respectively, Young's modulus, Poisson's ratio, and the thickness of the silicon substrate. $R_{0}$ and $R$ are the radii of curvature of the blank substrate (prior to the film deposition) and of the film/substrate composite, respectively.

\section{Results and Discussions}

The initial purpose of using the IBAD technique was to deposit a-C films under different pressure by simultaneously bombarding the film with a beam of argon atoms. Figure 1 shows the success of this procedure. It clearly demonstrates that the stress of the films could be varied (in the 1.5-4.5 GPa range) as a function of the bombardment energy (or assisting energy) with $\mathrm{Ar}^{+}$ions. The intrinsic compressive stress is attributed to the "knock-on" process on the carbon atoms due to intense bombardment by $\mathrm{Ar}^{+}$ions leading to higher stress. The "knock-on" process transforms $\mathrm{sp}^{2}$ into $\mathrm{sp}^{3}$ bonds, which is responsible for the increase in the compressive stress of the films [13, 18-20]. For energies above $100 \mathrm{eV}$, the stress reaches a limit with a subtle decrease trend towards higher energy due to the "thermal spike" effect, which promotes a distortion in the carbon matrix creating defects and consequent relaxation of the network.

The relative high stress indicates accumulated mechanical energy from the point of view of macroscopic properties. It 


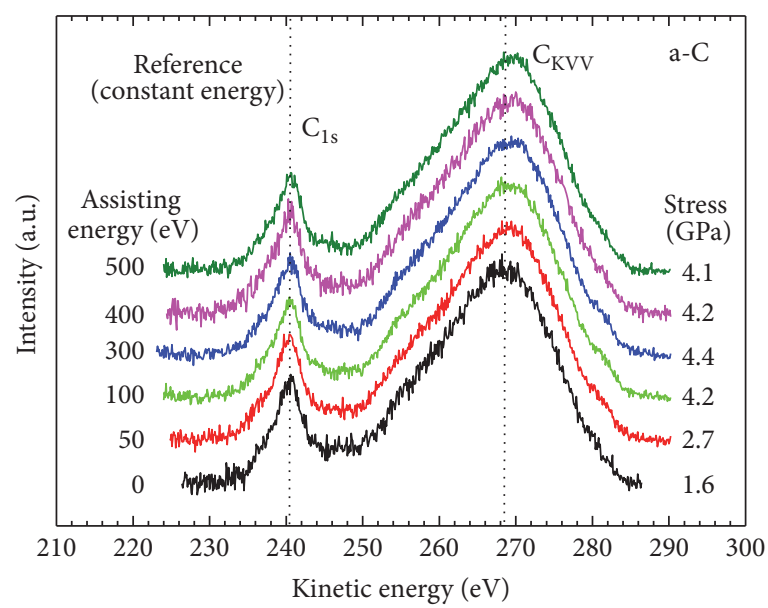

(a)

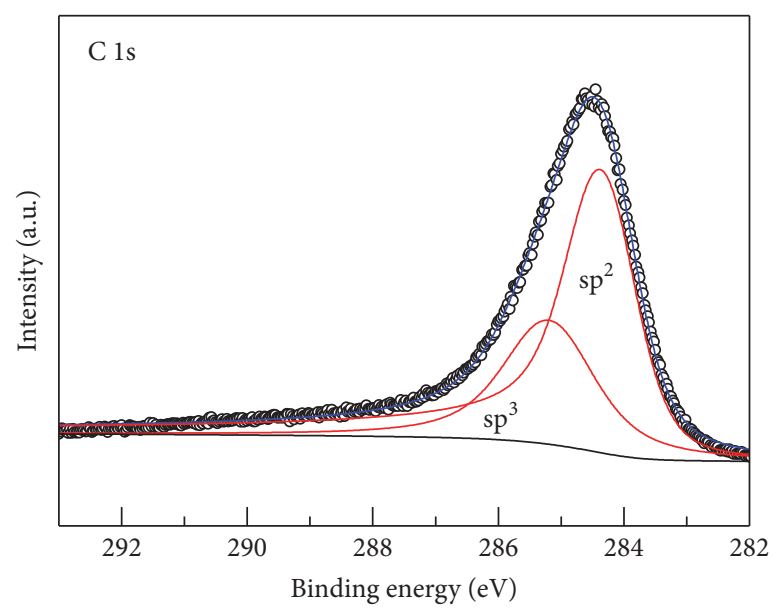

(b)

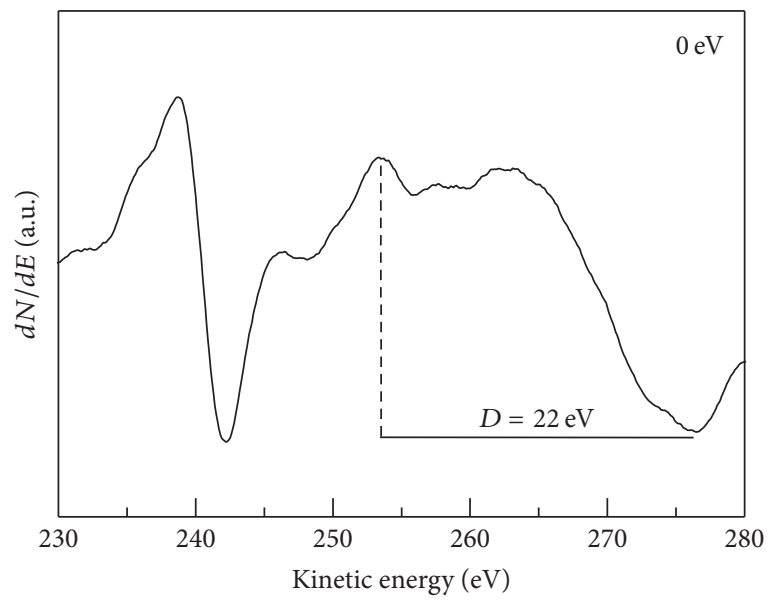

(c)

Figure 2: (a) XAES KVV spectra of carbon films. The background was subtracted out using the Shirley Model [16]. The very intense peak at approximately $240.5 \mathrm{eV}$ is due to excitation of $\mathrm{C} 1$ s core by the $\mathrm{K} \alpha$ of $\mathrm{O}$ adsorbed on the anode of the X-ray tube and is used as reference. The vertical lines are only guide for the eyes used to emphasize the energy shift of the $\mathrm{C}_{\mathrm{KVv}}$ 1s centroid from the $\mathrm{C}$ 1s core level used as constant energy reference. (b) XPS spectra showing the binding energy of the $\mathrm{Cls}$ core level excited by the $\mathrm{K} \alpha$ of $\mathrm{Al}$ and the contributions of sp ${ }^{2}$ and $\mathrm{sp}^{3}$ hybrid states. (c) First derivative spectra of the XAES KVV showing the D parameter estimated as $22 \mathrm{eV}$.

can be also understood, under the atomic point of view, that the increase of pressure, which concentrates the electronic cloud, is the result of the repulsion phenomenon between electrons. Thus, it is expected that the more external electronic states of the atoms feel a new type of interaction; that is, the band states manifest different behaviors under higher electronic density and it is expected to be reflected on Auger electrons. Figure 2(a) shows XAES spectra, as collected, with the background extracted by using the Shirley Model [16]. One can clearly observe that the $\mathrm{C}_{\mathrm{KVV}} 1 \mathrm{l}$ centroid energy is shifted to higher energy relative to $C_{1 s}$. Figure 2 (b) shows that the samples have high concentration of $\mathrm{sp}^{2}$ bonds. The parameter $D=22 \mathrm{eV}$ was also estimated for the purpose of characterizing the sample type (Figure 2(c)). It is known that the parameter $D$ for amorphous carbon films with graphite characteristics is approximately $21 \mathrm{eV}$ whereas for diamond type samples it is $14 \mathrm{eV}$ [2], indicating that our samples are highly graphitic.
Since the chemical environment of the a-C film does not change in the a-C film, we do not expect much change in the $\mathrm{C}$ 1s photopeak, Figure 2(a). However, the energy shift observed in the Auger electrons in Figure 2(a) hides details that are revealed by the second derivative method, as shown in Figure 3, adopting the method described in [6] but using the Savitzky-Golay smoothing procedure. These details reveal electronic transitions with more defined energy as seen by the presence of additional peaks in samples with higher bombardment energies, indicating atomic relaxation channels and uniformity of the $\mathrm{C}$ atoms in the matrix. Comparing Figure 3 with Figure 2(a), one can observe that the second derivative method brings much more details and information about the electronic structure of the film. In the following paragraphs let us examine the effect of the matrix pressure in the electronics levels. For that, we are focusing our discussion on the films prepared with low bias voltage (up to $100 \mathrm{~V}$ ), where one observes a significant increase of stress 


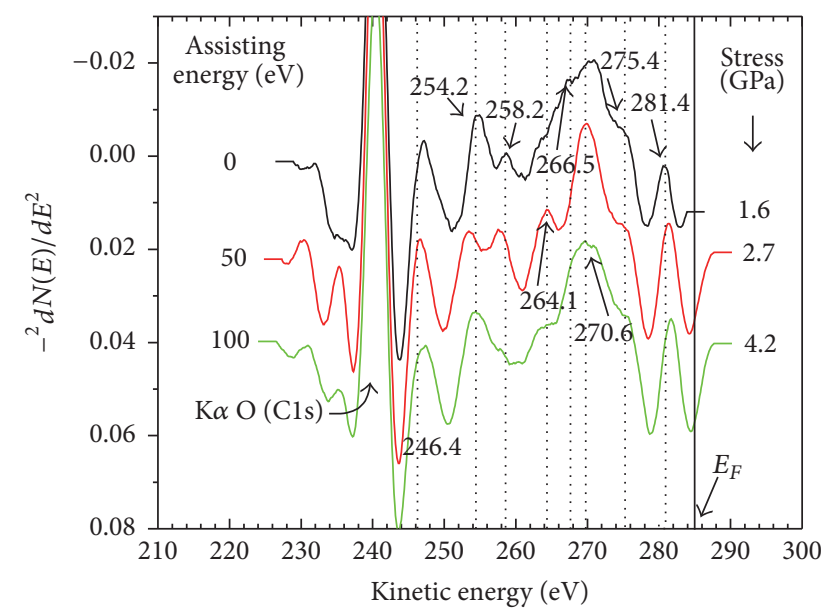

(a)

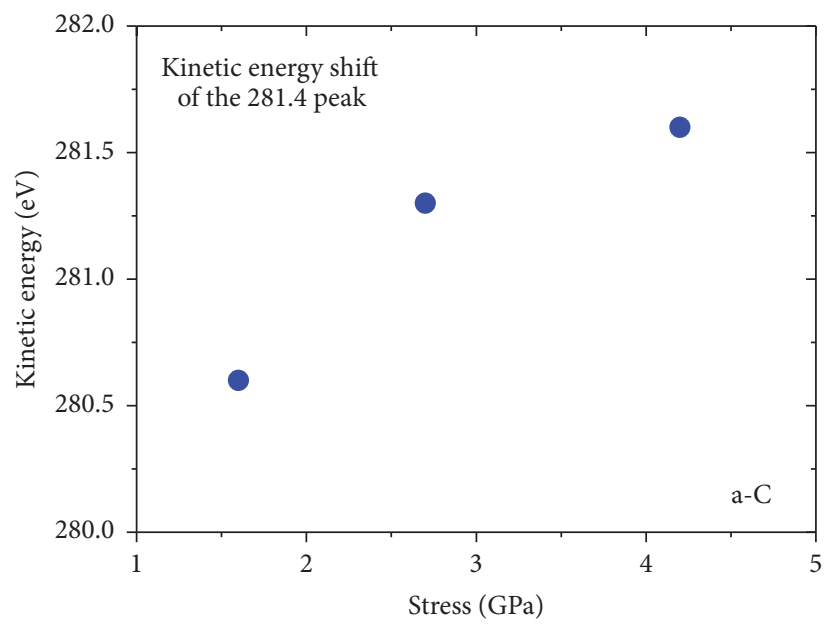

(b)

FIGURE 3: (a) Second derivative of the XAES KVV from a-C thin films with different pressure (stress). The Fermi energy was determined using C 1s in $284.3 \mathrm{eV}$. The very intense peak at approximately $240.5 \mathrm{eV}$ is due to excitation of C 1s core by $\mathrm{K} \alpha$ of $\mathrm{O}$ adsorbed on the anode of the X-ray tube. (b) Kinetic energy of the $p_{\pi}$ valence states as a function of the carbon film stress in amorphous carbon. The dotted lines are guides to the eyes.

due to the "know on" process, as mentioned above. For high bias voltage the "thermal spike" creates defects in the films, altering their structure.

First, a kinetic energy shift of the peak at approximately $281.4 \mathrm{eV}$ with increased stress is observed. This energy region has predominantly a $p_{\pi}$ character of valence states $[7,10-$ $12,14,15]$, which is a level close to the Fermi level, as indicated by the line in the figure. To better visualize this phenomenon Figure 3(b) shows that the kinetic energy has a tendency to grow with the stress of the film. Thus, the kinetic energy of this peak can be used to estimate the pressure of the carbon network. Another observation related to the $p_{\pi}$ valence states is that the intensity of the peak increases with stress and this is related to the number of occupied states due to the shift towards the Fermi level [21]. These are new effects not yet reported in literature, requiring extremely high stress, as the one achieved in this work $(4.5 \mathrm{GPa})$.

Figure 3 shows a peak at $275.4 \mathrm{eV}$, also associated with $p_{\pi}$ states, that has already been reported by the method of the second derivative $[6,7,10]$. Here we observe a slight tendency to increase with stress. This peak appears very intense in carbon nanotubes, and its presence is attributed to structural defects in the walls of the nanotubes. DOS calculations attribute this behavior to linkage between layers [11].

The peaks appearing at $\sim 270.6 \mathrm{eV}, 266.5 \mathrm{eV}$, and $264.1 \mathrm{eV}$ are predominantly associated with $p_{\sigma}$ states [7]. Note that they are not clearly resolved in the film with the lowest stress $(1.6 \mathrm{GPa})$, which was prepared without argon bombardment. They become apparent for different argon assisted energy. The peaks at $258,2 \mathrm{eV}$ and $254,2 \mathrm{eV}$ have been associated with hybrid states of sp character or dangling bonds [7]. We observe some change in the intensity and positon, not clearly related to the stress. Finally, in relation to the deeper s-type states, there is a peak at $246.4 \mathrm{eV}$, which is not significantly altered with stress and has the same intensity at the same energy in each of the samples and may be a characteristic peak of amorphous carbon, given by a miscellany between states formed by graphite and diamond bonds [15]

In summary, it is observed that, in the KVV spectrum range, using the second derivative method, the most external electronic states (above $264.1 \mathrm{eV}$, i.e., $p_{\pi}$ and $p_{\sigma}$ states) are more influenced by stress than the sp hybrid states.

\section{Conclusion}

Carbon thin films under different internal pressures (compressive stress) were deposited by ion beam deposition and simultaneously bombarded with a beam of $\mathrm{Ar}^{+}$ions. Using this technique it was possible to prepare films at extremely high stress in the $1.5-4.5 \mathrm{GPa}$ range. The XAES technique permitted the observation of the influence that stress causes to the density of states. The energy shift of the Auger peak to higher energy as the stress increases also increases the density of electrons at energies closer to the Fermi level. Using the second derivative of XAES KVV carbon spectra, we found details which allowed the observation of variations on density of states as a result of the high compressive intrinsic stress. The increase in the population of states near the Fermi level can drive interest in the production of a-C films with high stress, for instance, to improve the electron field emission. The line shape of the XAES and the detailed structure obtained through the second derivative method can be used as characteristic patterns of $\mathrm{a}-\mathrm{C}$ as has been done for other carbon based films.

\section{Conflicts of Interest}

The authors declare that there are no conflicts of interest regarding the publication of this paper. The mentioned received funding in the Acknowledgments does not lead to 
any conflicts of interest regarding the publication of this manuscript.

\section{Acknowledgments}

This work was supported by the following Brazilian research finance agencies: CAPES, CNPq, FAPESP, and INES/INCT. The authors also acknowledge Lamult/UNICAMP/IFGW.

\section{References}

[1] R. G. Lacerda, M. C. dos Santos, L. R. Tessler, P. Hammer, F. Alvarez, and F. C. Marques, "Pressure induced physical changes of noble gases implanted in highly stressed amorphous carbon films," Physical Review B, vol. 68, no. 5, Article ID 054104, 2003.

[2] J. Zemek, J. Zalman, and A. Luches, "XAES and XPS study of amorphous carbon nitride layers," Applied Surface Science, vol. 133, no. 1-2, pp. 27-32, 1998.

[3] F. C. Marques, R. G. Lacerda, M. M. de Lima Jr., and J. Vilcarromero, "Hard a-C:H films deposited at high deposition rate," Thin Solid Films, vol. 343-344, no. 1-2, pp. 222-225, 1999.

[4] B. Lesiak, P. Mrozek, A. Jablonski, and A. Jóźwik, "Analysis of the Auger KLL spectra of carbon by the pattern recognition method," Surface and Interface Analysis, vol. 8, no. 3, pp. 121126, 1986.

[5] Y. Mizokawa, T. Miyasato, S. Nakamura, K. M. Geib, and C. W. Wilmsen, "Comparison of the CKLL first-derivative auger spectra from XPS and AES using diamond, graphite, Si/C and diamond-like-carbon films," Surface Science, vol. 182, no. 3, pp. 431-438, 1987.

[6] J. C. Lascovich and A. Santoni, "Study of the occupied electronic density of states ofcarbon samples by using second derivative carbono KVV Auger spectra," Applied Surface Science, vol. 103, no. 3, pp. 245-253, 1996.

[7] J. C. Lascovich and V. Rosato, "Analysis of the electronic structure of hydrogenated amorphous carbon via Auger spectroscopy," Applied Surface Science, vol. 152, no. 1, pp. 10-18, 1999.

[8] A. P. Dementjev, A. de Graaf, D. I. Dolgiy, E. D. Olshanski, Y. M. Shulga, and A. A. Serov, "CNx film characterization by surface sensitive methods: XPS and XAES," Diamond and Related Materials, vol. 8, no. 2-5, pp. 601-604, 1999.

[9] G. Speranza, L. Calliari, N. Laidani, and M. Anderle, "Semiquantitative description of $C$ hybridization via s- and $\mathrm{p}$-partial density of states probing: an electron spectroscopy study," Diamond and Related Materials, vol. 9, no. 11, pp. 1856-1861, 2000.

[10] J. C. Lascovich, V. Rosato, and A. Santoni, "Study of the microscopic scale structure of carbon-based materials: a comparison between Auger-derived density of states structures and molecular dynamics simulations," Surface Science, vol. 467, no. 1-3, pp. 139-151, 2000.

[11] I. P. Asanov, L. G. Bulusheva, and A. V. Okotrub, "XAES study of carbon fluoride and carbon materials," Journal of Electron Spectroscopy and Related Phenomena, vol. 114-116, pp. 243-249, 2001.

[12] L. Calliari, G. Speranza, J. C. Lascovich, and A. Santoni, "The graphite core-valence-valence Auger spectrum," Surface Science, vol. 501, no. 3, pp. 253-260, 2002.

[13] W. Lu and K. Komvopoulos, "Effect of stress-induced phase transformation on nanomechanical properties of sputtered amorphous carbon films," Applied Physics Letters, vol. 82, no. 15, pp. 2437-2439, 2003.
[14] J. E. Houston, J. W. Rogers Jr., R. R. Rye, F. L. Hutson, and D. E. Ramaker, "Relationship between the Auger line shape and the electronic properties of graphite," Physical Review B, vol. 34, no. 2, pp. 1215-1226, 1986.

[15] K. Endo, S. Koizumi, T. Otsuka et al., "Analysis of electron spectra of carbon allotropes (diamond, graphite, fullerene) by density functional theory calculations using the model molecules," Journal of Physical Chemistry A, vol. 107, no. 44, pp. 9403-9408, 2003.

[16] D. A. Shirley, "High-resolution X-ray photoemission spectrum of the valence bands of gold," Physical Review B, vol. 5, no. 12, pp. 4709-4714, 1972.

[17] M. M. de Lima Jr., R. G. Lacerda, J. Vilcarromero, and F. C. Marques, "Coefficient of thermal expansion and elastic modulus of thin films," Journal of Applied Physics, vol. 86, no. 9, pp. 4936-4942, 1999.

[18] Y. Lifshitz, G. D. Lempert, E. Grossman et al., "Growth mechanisms of DLC films from $\mathrm{C}^{+}$ions: experimental studies," Diamond and Related Materials, vol. 4, no. 4, pp. 318-323, 1995.

[19] E. F. Motta, G. A. Viana, D. S. Silva, A. D. S. Côrtes, F. L. Freire Jr., and F. C. Marques, "Structural, surface and thermomechanical properties of intrinsic and argon implanted tetrahedral amorphous carbon," Journal of Vacuum Science and Technology A: Vacuum, Surfaces and Films, vol. 31, no. 2, Article ID 021502, 2013.

[20] F. C. Marques, G. A. Viana, E. F. Motta et al., "Argon implantation in tetrahedral amorphous carbon deposited by filtered cathodic vacuum Arc," Journal of Materials Engineering and Performance, vol. 22, no. 5, pp. 1396-1404, 2013.

[21] V. V. Khvostov, M. B. Guseva, V. G. Babaev, and O. Y. Rylova, "Auger-spectroscopy studies of the electronic structure of amorphous," Surface Science, vol. 169, no. 1, pp. L253-L258, 1986. 

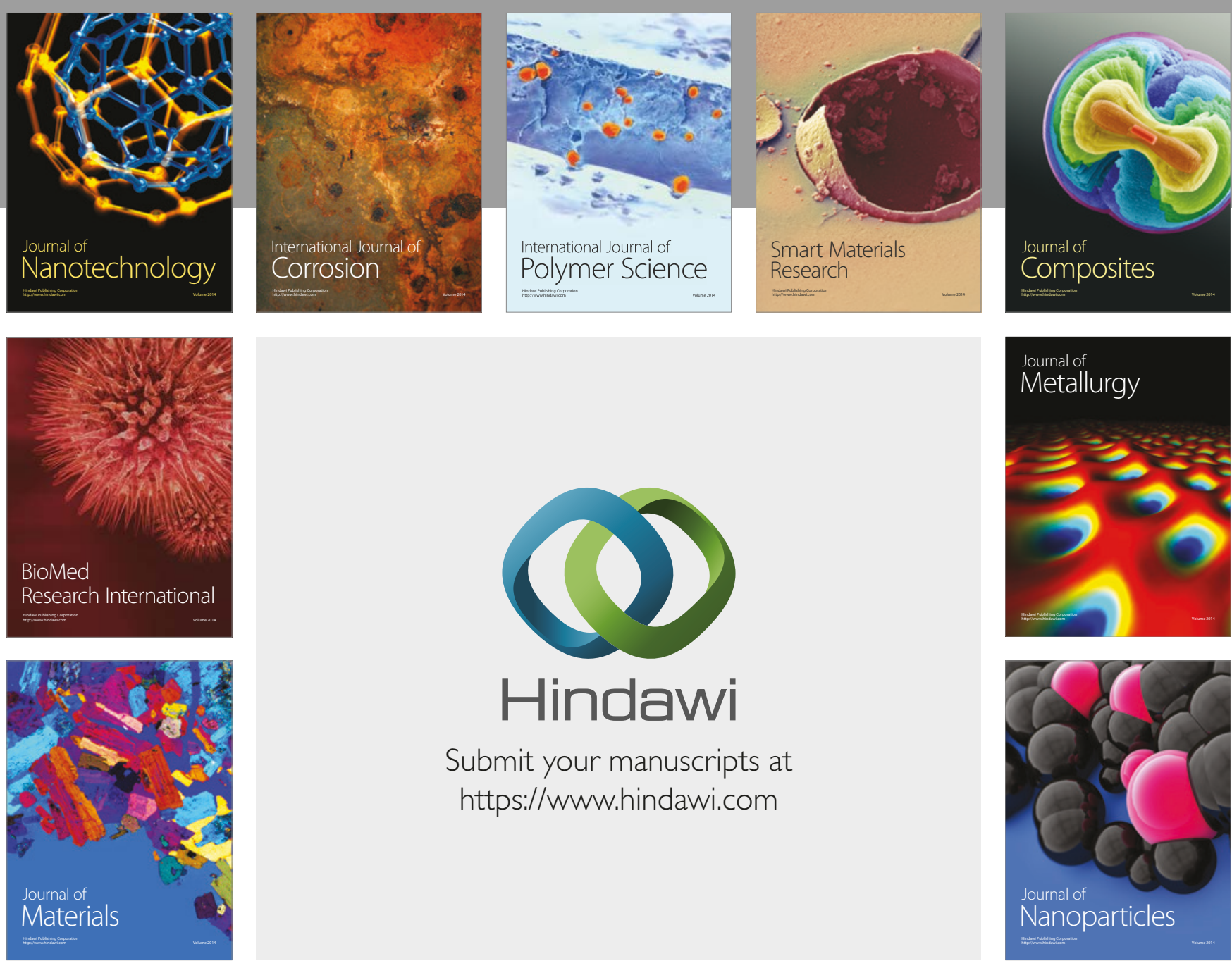

\section{Hindawi}

Submit your manuscripts at

https://www.hindawi.com
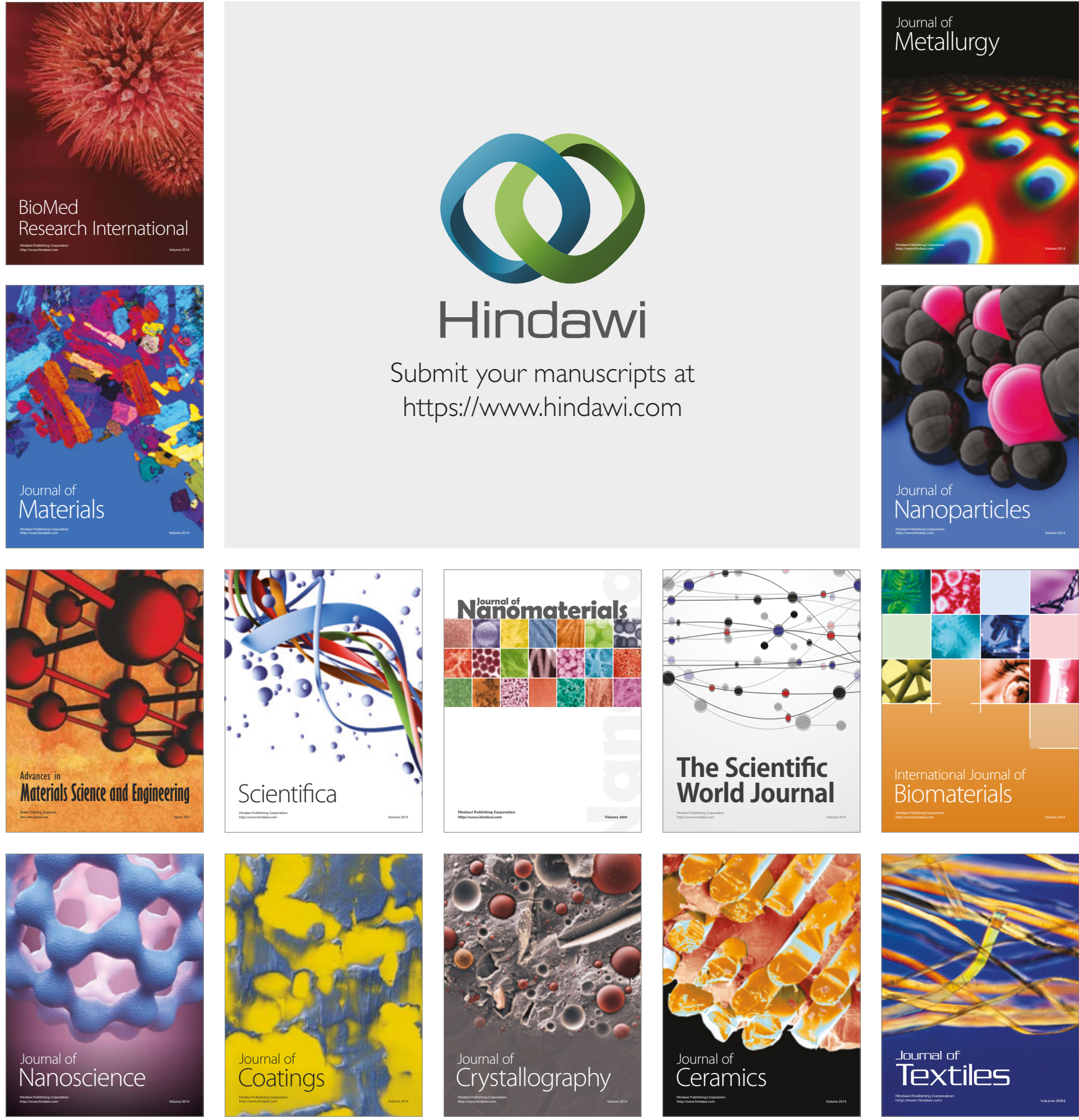

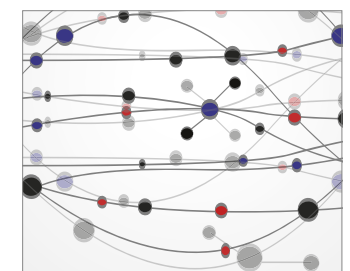

The Scientific World Journal
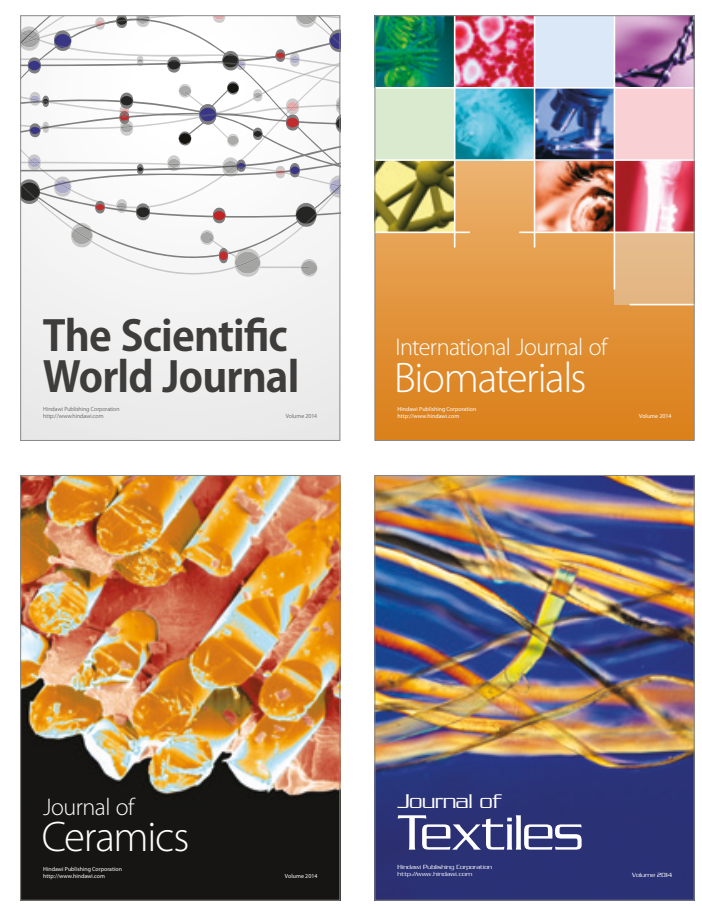